\title{
Formation of the regularly arranged tubular pores during electrophoretic deposition
}

\author{
Cong Lin ${ }^{1,2, *}$, WuXin Li ${ }^{1}$, CengCeng FAng ${ }^{1}$, Qidan Wu ${ }^{1}$, Hucheng Xie $^{1}$, \\ DELIANG XU ${ }^{1}$, BIZHI $\mathrm{XU}^{3}$ \\ ${ }^{1}$ School of Material Science and Engineering, Fuzhou University, Fuzhou 350108, People's Republic of China \\ ${ }^{2}$ Key Laboratory of Eco-materials Advanced Technology, Fujian Province University, Fuzhou 350108, \\ People's Republic of China \\ ${ }^{3}$ Department of Materials Engineering, Zhicheng College, Fuzhou University, Fuzhou 350002, People's Republic of China
}

\begin{abstract}
The formation mechanism of regularly arranged tubular pores during an acid-based electrophoretic deposition (EPD) process was explored by studying the influence of gauze electrodes and suspension properties on the pore structures. The gauze electrodes can change the intensity of electrical field on the electrode surface, and thus control the pore locations. The mesh size not only restricts the ultimate pore size, but also determines the regularity of the pores. Under specific experimental conditions, a critical value of mesh size for attaining the regularity of the pores arrangement can be determined. Meanwhile, the pore structures can be controlled by a combination of $\mathrm{pH}$ value and zeta potential of the suspensions. The strength of the acidity is also one of the determinants to the final structures.
\end{abstract}

Keywords: arranged tubular pores; electrophoretic deposition; suspension properties; alumina porous ceramics

(C) Wroclaw University of Science and Technology.

\section{Introduction}

Tubular porous ceramics are attracting more and more attention in the application of insulations, filters, heat exchangers and adsorbent carriers $[1,2]$. The combustion or volatilization of polymer media is one of the typical methods to prepare the tubular porous ceramics. For preparing regularly arranged tubular pores, the extrusion method is always recommended in the industrial field. However, these methods are complicated, expensive, and time consuming due to the necessity of template removing or the molding process [3-5].

The tubular porous ceramics can also be fabricated by a combination of aqueous-based electrophoretic deposition (EPD) process with simultaneous gas generation from water electrolysis [6-9]. Electrophoretic deposition has been proved

*E-mail: lincong@fzu.edu.cn to be a very efficient method in producing dense green bodies [10, 11], laminates [12-15], composites [16-18] and coatings [19-21] for many ceramics categories. For thick deposit applications, aqueous based electrophoretic deposition is often questioned due to gas generation by the electrolysis of water, which may result in the unexpected pore structures [22]. In most cases this phenomenon need to be avoided, but it can be quite useful to produce the tubular porous ceramics without any molding or template removing processes. Furthermore, when using the gauze electrodes instead of flat plate electrodes, the regularly arranged tubular pores can also be produced by the aqueous based electrophoretic deposition [7, 10]. Moritz et al. [7, 8, 10] have tried this method in several kinds of ceramics in platinum gauze electrodes, and successfully obtained regularly arranged tubular pores in the locations of the mesh holes. They also found that the mesh sizes had great influence on the distribution and regularity of the pores [7]. Since it is sure enough that 
the pores are produced by the electrolytic gas generation in aqueous suspensions, the reason why the gauze electrodes can make them arrange regularly is still not explained well. To clarify this, the factors that might affect the formation of the regularly arranged tubular pores, such as mesh size and suspension properties need further discussion. In this paper, the electric field distributions in the gauze electrode during electrophoretic deposition was calculated for different mesh sizes. The effects of the suspension properties on the formation of the tubular pores were also deeply discussed. After that, a probable formation model was set up, which tries to explain the forming process of the regularly arranged pores.

\section{Materials and experiments}

A commercially available alumina powders (Demeter (Suzhou) Electronics Environmental Materials Co., Ltd.) having $120 \mathrm{~nm}$ average particle size were used for preparing the suspensions. Several acid solutions, such as carboxylic acid with different numbers of methyl groups, hydrochloric acid and nitric acid were used as solvents with designed concentrations. The solid content in the suspension was set as $15 \mathrm{wt} \%$. The suspension was magnetically stirred for 2 hours followed by 15 min ultrasonic treatment. The zeta potential of each suspension was measured by a zeta potential instrument (JS94H). The electrophoretic deposition was conducted using a continuous DC power source (DYY-6D, Liuyi Instrument Plant, Beijing) with stainless steel as electrodes. No obvious corrosion could be observed on the surface of the stainless steel electrodes in the current experimental conditions with limited acidity and deposition time. The dimensions of the deposition electrodes were $20 \mathrm{~mm}^{2} \times 20 \mathrm{~mm}^{2}$, and the distance between the two electrodes was adjusted to be $20 \mathrm{~mm}$. A piece of stainless steel gauze was assembled between the two electrodes and was electrically connected with the cathode, which was the deposition electrode. The square-shape meshes with different dimensions from $0.1986 \mathrm{~mm}$ to $0.6567 \mathrm{~mm}$ were used. Electrophoretic deposition without a gauze was also performed for comparison. The applied constant voltages during electrophoretic deposition were set from $10 \mathrm{~V}$, and the deposition time for each experiment was $10 \mathrm{~min}$. The pore structure of the green bodies after electrophoretic deposition was investigated by optical microscopy (BLSZM745, Belona). The XRD patterns (Fig. 1) of the dried green samples confirm that their chemical compositions have not changed during the electrophoretic deposition. The average pore sizes and distributions were investigated by measuring more than 100 isolated pores.

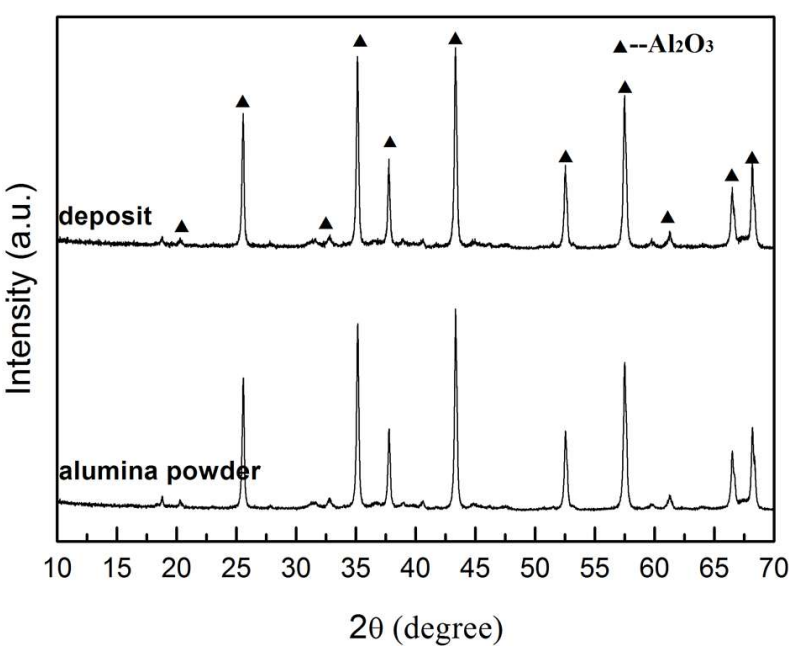

Fig. 1. XRD pattern of the deposited alumina powders and green body deposit.

\section{Results and discussion}

The optical photographs of the green body deposited using different gauze sizes are shown in Fig. 2. The pore sizes of the green bodies have been determined from these photographs and the results are exhibited in Table 1. Tubular pores can be formed without the assistance of any gauze, and randomly arranged cylindrical pores were formed by random bubble collapse during the deposition process. A critical value for the current experimental conditions can be assumed when the coalescent bubbles burst through the deposit. Fig. 2b to Fig. 2e show the photos of the green bodies prepared on the gauze surfaces with various mesh sizes. The final pore sizes seem to be increasing linearly with the mesh size which indicates that 
the pores were grown under the restriction of the mesh grid. However, the green body produced by the grid with $0.1985 \mathrm{~mm}$ in size has not generated regularly arranged pore structures, but randomly distributed pores instead. For comparison, all the grids that were larger in size than the critical value showed a potential to form regularly arranged tubular pores. On the other hand, the ratio of the pore size to the mesh size $\left(\mathrm{S}_{\mathrm{p}} / \mathrm{S}_{\mathrm{m}}\right)$ was found to be decreasing, which means that the restriction of the grids on the pores coalescence was reduced with the increasing mesh size. The critical value of the grid size is when the $S_{p} / S_{m}$ value is very close to 1 , which could be defined as the breakthrough point whether the pore structure is regularly arranged or not. When the $S_{p} / S_{m}$ value is greater than this breakthrough point, it would happen that the growth of the bubbles on the electrode surface would be restricted by the grid. These partly developed bubbles would not be stable and could undergo a further coalescence process after escaping from the grid. In this sense, the coalescence of these small bubbles can not only enlarge the pore size, but also disorganize the arrangement order. As a result, the irregularly arranged pores could be observed when the mesh size is smaller than the critical pore size.

Table 1. The average values of pores size and the ratio of the pore size to the mesh size $\left(\mathrm{S}_{\mathrm{p}} / \mathrm{S}_{\mathrm{m}}\right)$.

\begin{tabular}{ccc}
\hline Mesh size [mm] & Average pore sizes $[\mathrm{mm}]$ & $\mathrm{S}_{\mathrm{p}} / \mathrm{S}_{\mathrm{m}}$ \\
\hline \hline 0.6567 & $0.5834 \pm 0.1148$ & 0.88933 \\
0.4650 & $0.4536 \pm 0.1124$ & 0.97548 \\
0.3033 & $0.3110 \pm 0.0840$ & 1.02539 \\
0.1985 & $0.2483 \pm 0.1251$ & 1.25088 \\
without mesh & $0.2819 \pm 0.1015$ & - \\
\hline
\end{tabular}

Since the relationship between the pore dimensions and mesh size has been clarified according to the performed discussion, the reason why the pores can always be located inside the grids is still ambiguous. It is well known that the pore evolution on the electrode surface must be determined by the deposition process and water electrolysis, both of which are relevant to the electric field intensity on the electrode surface. In common sense, the increase in the electric field intensity will effectively accelerate both deposition and electrolysis processes. The distribution of electric field on the deposited electrodes is shown in Fig. 3. The electric field intensity differences between the mesh grids and mesh holes are also shown in Table 2. It is not difficult to find from the calculation results that the intensities of the electric field increased with the mesh sizes, not only on the mesh grid but also inside the mesh holes. And a more important fact is that the electric field intensity on the surface of the mesh grid is much higher than that inside the mesh holes. Based on this observation, the deposition rate of the alumina particles is much higher, and consequently thicker and denser deposit is produced at the location of the mesh grid than inside the mesh holes. Likewise, it seems that the electrolysis on the grid must also be accelerated and produce initially more bubbles on the grid surface. However, the generation of these bubbles would not last long because the dense deposit is formed on the grid surface very quickly, and then only few water available for electrolysis might remain on the grid surface to retain the electrolysis reaction. On the other hand, relatively loose deposit is easily destroyed inside the mesh holes, where the plate electrodes have positive effect on the coalescence of the bubbles to reach their critical size due to the thermocapillary flow and migration [23]. As a result, the constantly released hydrogen gas bubbles generate inside the mesh holes, where with a great probability, the pores finally develop.

Table 2. The differences of the electric field intensities between the mesh grids and holes in the gauzes with different mesh sizes.

\begin{tabular}{cccc}
\hline Mesh & \multicolumn{3}{c}{ Electric field intensity [V/mm] } \\
\cline { 2 - 4 } size $[\mathrm{mm}]$ & Mesh grid/E & Mesh hole/ $/ \mathrm{E}_{\mathrm{h}}$ & $\Delta \mathrm{E}=\mathrm{E}_{\mathrm{g}}-\mathrm{E}_{\mathrm{h}}$ \\
\hline \hline 0.6567 & 1283.7 & 522.66 & 761.04 \\
0.465 & 1201.8 & 414.04 & 787.76 \\
0.3033 & 1191.6 & 382.67 & 808.93 \\
0.1985 & 1080.2 & 198.74 & 881.46 \\
\hline
\end{tabular}

As already mentioned, although the mesh grids can differentiate electric field intensities on the surface of the electrode and help to determine the pore 


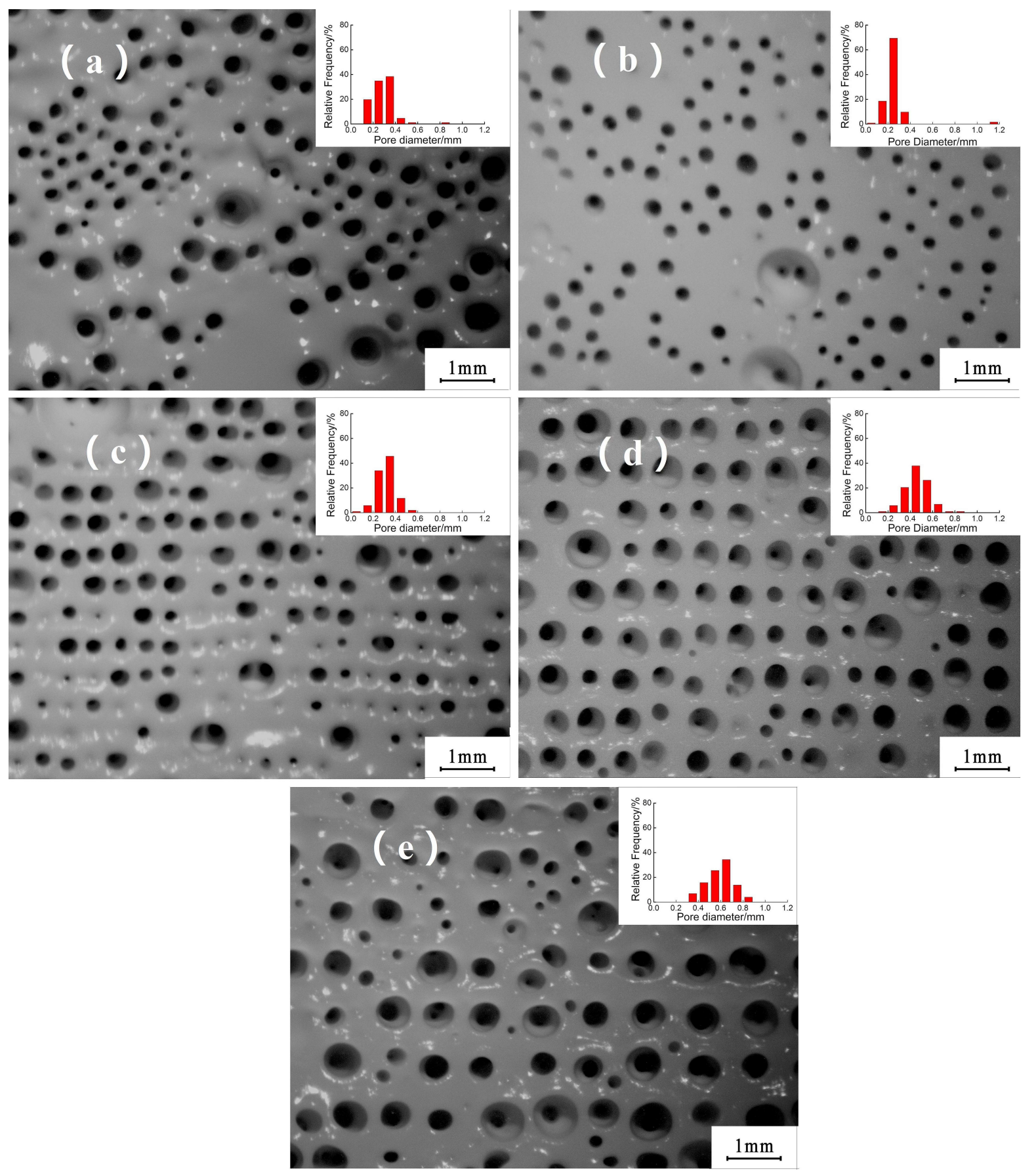

Fig. 2. Optical photographs of the tubular porous alumina ceramics prepared (a) without a mesh; and at various mesh sizes: (b) $0.1985 \mathrm{~mm}$; (c) $0.3033 \mathrm{~mm}$; (d) $0.4650 \mathrm{~mm}$; (e) $0.6567 \mathrm{~mm}$.

locations, regularly arranged tubular pores have not value was set as 4.13 , the suspension containing been created in any kind of acid-based suspension hydrocholoric acid or nitric acid has not resulted systems. Fig. 4 shows the optical photographs of in a porous appearance after EPD, but a smooth the deposited green body from alumina suspen- and flat surface instead. We can find the arranged sions with different types of acids. When the $\mathrm{pH}$ pores situated at the site close to the electrode, as 

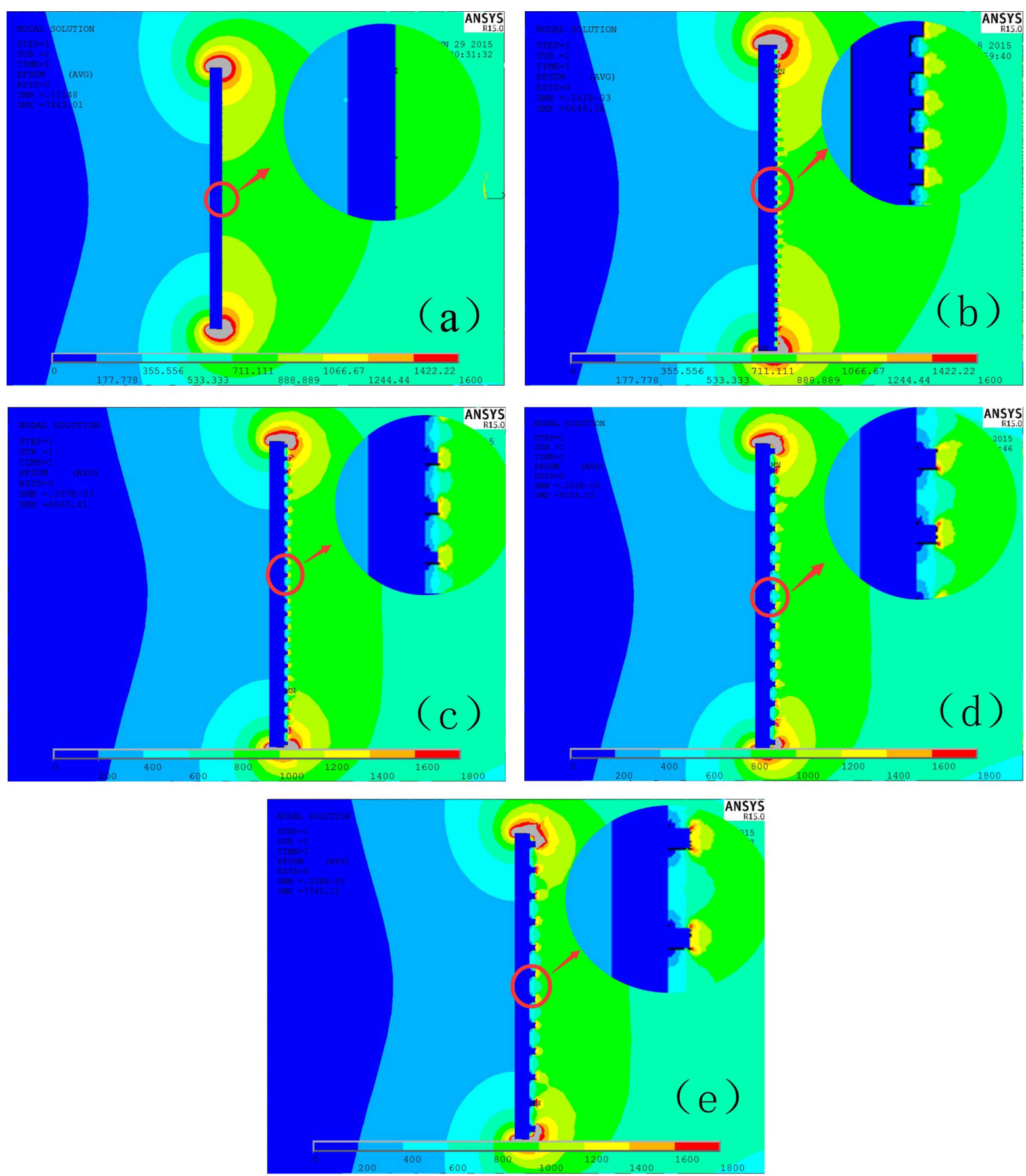

Fig. 3. Calculated electric field strength distribution on the gauze surface for different mesh sizes (a) no mesh; (b) $0.1985 \mathrm{~mm}$; (c) $0.3033 \mathrm{~mm}$; (d) $0.4650 \mathrm{~mm}$; (e) $0.6567 \mathrm{~mm}$.

shown in the inset of Fig. 4b. These buried pores are covered deeply by the deposit and have the size equal to the mesh dimension. When the $\mathrm{pH}$ value was decreased by adding more hydrochloric acid, regularly arranged tubular pores have been formed, though the increased acid content (Fig. 4d) has provoked some uncontrollable coalescence and broke the regularity of the pore arrangement.

The competition between deposition rate and bubble release degree ultimately determine 


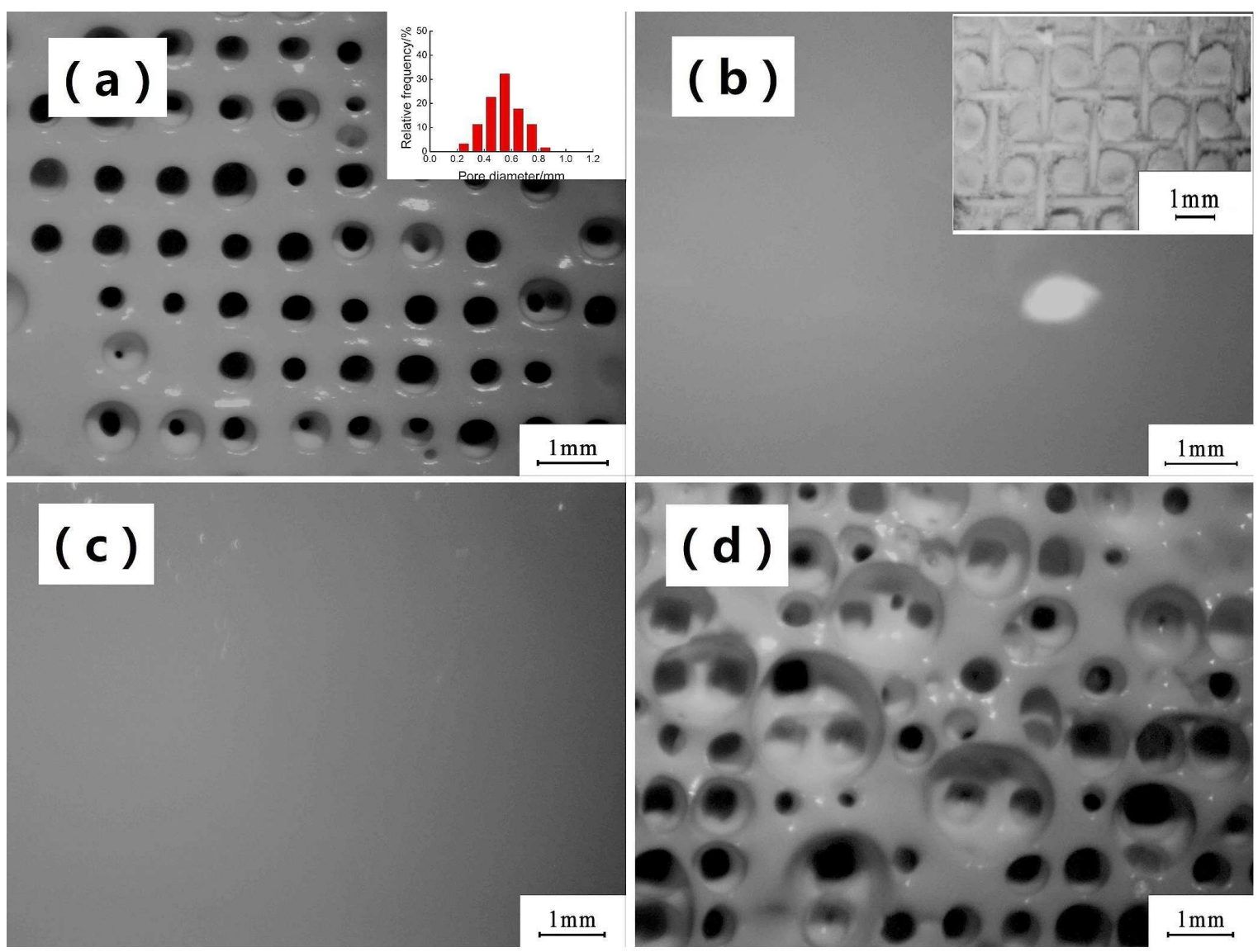

Fig. 4. Optical photographs of surfaces with a deposit from suspensions containing different types of acids: (a) propionic acid, $\mathrm{pH}=4.13$; (b) hydrochloric acid, $\mathrm{pH}=4.12$; (c) nitric acid, $\mathrm{pH}=4.13$; (d) hydrochloric acid, $\mathrm{pH}=2.78$. The insets represent (a) the pore diameter distribution determined from the relevant optical photographs; (b) the optical photograph of the opposite surface (close to the gauze) of the green body.

whether the pore structure is regular. In order to explain the effect of hydrogen ions concentration on the pore formation during electrophoretic deposition, the variation of $\mathrm{pH}$ values of the suspension along with the deposition time were recorded in Fig. 5. In the suspension whose original $\mathrm{pH}$ value was 4.13 , either in the hydrocholoric or propionic acid, no significant changes could be found during the procedure of EPD. According to the optical photographs, the propionic acid might ionize continuously to produce hydrogen ions in order to compensate their consumption during the formation of hydrogen gas. In this sense, the hydrogen ions concentration is kept constant in propionic acid based suspension. In the system with hydrocholoric acid, the deposit structure had a great effect on the $\mathrm{pH}$ value modification. When the porous deposit was produced, no more hydrogen ions could be generated due to the complete ionization of $\mathrm{HCl}$, so that the $\mathrm{pH}$ values would consequently increase during the EPD process. As shown in Fig. 5, the $\mathrm{pH}$ value increased from 2.78 to 3.10 when the hydrocholoric acid was used as an acid source. However, because the reduction reaction between hydrogen ions and electrons could only happen on the surface of the electrode, no more hydrogen gas was generated after the bubbles were covered by the deposit, which resulted in the stabilization of the $\mathrm{pH}$ value during the electrophoretic deposition in the hydrocholoric acid suspension with lower acid concentration.

In order to deeply clarify the relationship between the pore structures and the suspension 


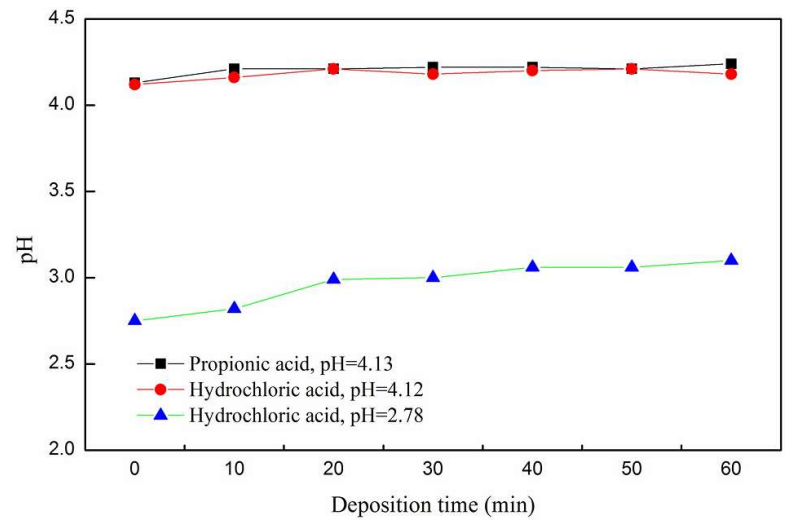

Fig. 5. The $\mathrm{pH}$ values variation during the deposition from suspensions with different acids.

properties with acid addition, further studies of EPD were performed with diverse acid categories and concentrations. The structures of the deposits were statistically classified on the basis of the $\mathrm{pH}$ values and zeta potentials of the suspension, which represent the gas release rate and the particle deposition rate, respectively. The acid categories included carboxylic acid (formic acid, acetic acid, and propionic acid), hydrochloric acid and nitric acid as well. As shown in Fig. 6, a sufficiently high value of zeta potential was the prerequisite for the formation of the tubular pore structure during EPD. Very few deposit could be produced below the zeta potential value of 20 because of the lower surface charge and resulting aggregation. For the suspensions with higher zeta potentials, three different pore structures can be distinguished depending on the $\mathrm{pH}$ values and the acid types: (i) $\mathrm{pH}<3.6$ in all kinds of acids: as shown in the inverted triangle region in Fig. 6, irregularly oriented pores can be achieved under this condition because of the fast release rate and uncontrollable coalescence of bubbles due to the higher concentration of hydrogen ions; (ii) $\mathrm{pH}>3.6$ in strong acids: the oriented pores are covered by quickly densified deposit (the hollow triangles), and can no longer release hydrogen gas due to the depletion of hydrogen ions close to the electrode; (iii) $\mathrm{pH}>3.6$ in weak acids: the regularly arranged tubular pores can be formed under such conditions. The existence of the weak acids contributes to the steady release of hydrogen ions, forming gas bubbles which can break through the deposit layer at its relatively weak part, for example, the position of mesh holes, and eventually form the bubble channels inside the deposit.

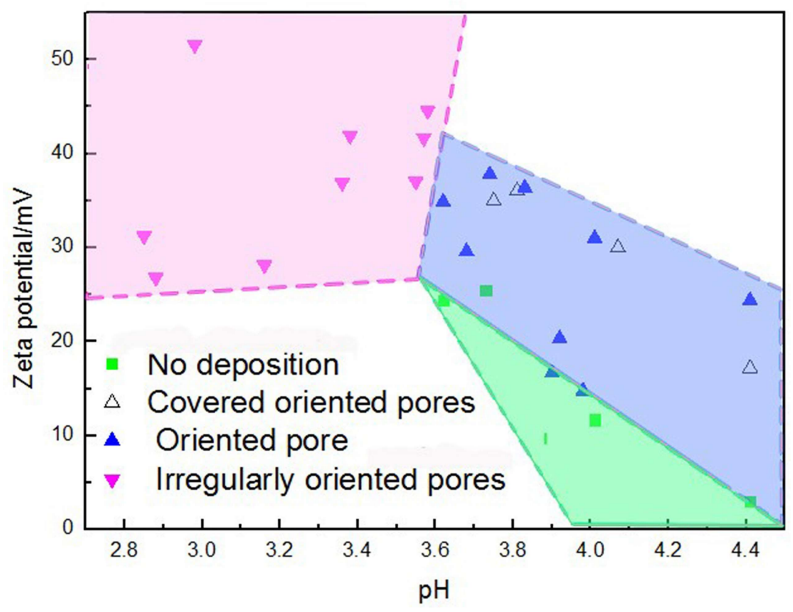

Fig. 6. Effect of the $\mathrm{pH}$ values and zeta potential on the deposited pore structures.

\section{Conclusions}

The deposits with regularly arranged tubular pores can be successfully prepared by an acid-based electrophoretic deposition. The roles of the gauze electrodes and suspension properties on the final pore structures have been deeply studied in this work. Different intensities of electric field on the mesh grid and inside the mesh holes are the primary reason that the pores can be arranged regularly with the help of a gauze electrode. A critical value of the mesh size can be found in specific conditions, and orderly distributed unidirectional pores can be achieved when the mesh sizes are beyond this value. The competition between the deposition rate and bubble release extent, together with the control of bubble coalescence, determine the final pore structures.

\section{Acknowledgements}

The project is supported by the National Natural Science Foundation of China (No. 51102046, No. 51602055) and the Natural Science Foundation of Fujian Province (No. 2013J05067). The authors would also like to thank for the "Qishan Scholar" Scientific Research Startup Project of Fuzhou University (No. 650338) and the financial support (No. ZJ1438) from the Zhicheng College of Fuzhou University. 


\section{References}

[1] Zhu Z., Xiao J., He W., Wang T., Wei Z., Dong Y., J. Eur. Ceram. Soc., 35 (2015), 3187.

[2] Hedfi I., Hamdi N., Rodriguez M.A., Srasra E., Ceram. Int., 42 (2016), 5089.

[3] Popa C., OKayasu Y., Katsumata K.-I., Isobe T., Matsushita N., Nakajima A., Kurata T., OKADA K., J. Mater. Sci., 48 (2013), 941.

[4] Moon Y.-W., Shin K.-H., KoH Y.-H., Yook S.-W., HAN C.-M., KIM H.-E., J. Am. Ceram. Soc., 95 (2012), 1803.

[5] Al-Harbi O.A., ÖZgür C., Khan M.M., Ceram. Int., 41 (2015), 3526.

[6] Hamagami J.-I., Ato Y., Kanamura K., Solid State Ionics, 172 (2004), 331.

[7] Moritz K., Aneziris C.G., Adv. Eng. Mater, 13 (2011), 82.

[8] Moritz K., BallaschK U., Schmidt G., HubÁlková J., Aneziris C.G., J. Eur. Ceram. Soc., 36 (2016), 333.

[9] Nakahira A., Nishimura F., Kato S., Iwata M., TAkeda S., J. Am. Ceram. Soc., 86 (2003), 1230.

[10] Moritz K., Aneziris C.G., J. Eur. Ceram. Soc., 34 (2014), 401.

[11] Abdelkader A.M., Garcia S.J., ZwaAg S.V.D., Ceram. Int., 39 (2013), 3429.

[12] Dzepina B., Sigalas I., Herrmann M., NiLEN R.,, Int. J. Refract. Met. H., 36 (2013), 126.
[13] Li Y., Gao J., Yang J., Wang H., Qiao G., Key Eng. Mater, 368 - 372 (2008), 1841.

[14] Hadraba H., Drdlik D., Chlup Z., Maca K., Dlouhy I., Cihlar J., J. Eur. Ceram. Soc., 32 (2012), 2053.

[15] Hadraba H., Drdlik D., Chlup Z., Maca K., Dlouhy I., Key Eng. Mater., 507 (2012), 209.

[16] Stojanovic D., Jokic B., Veljovic D., PetroVIC R., Uskokovic P.S., JANACKOVIC D., J. Eur. Ceram. Soc., 27 (2007), 1595.

[17] Olevsky E.A., Wang X., Maximenko A., MeyERs M.A., J. Am. Ceram. Soc., 90 (2007), 3047.

[18] LU X.J., Xiao P., J. Eur. Ceram. Soc., 27 (2007), 2613.

[19] Corni I., Neumann N., Novak S., König K., Veronesi P., Chen Q., Ryan M.P., Boccaccini A.R., Surf. Coat. Tech., 203 (2009), 1349.

[20] Baufeld B., Ratzer-Scheibe H.-J., Biest VAN DER O., Adv. Mater. Res., 75 (2009), 47.

[21] Rial L., Rodal P., Lopez-Alvarez M., Borrajo J.P., Solla E., Serra J., Gonzalez P., LeON B., Mater. Sci. Forum, 587 - 588 (2008), 86.

[22] Besra L., LiU M., Prog. Mater. Sci., 52 (2007), 1.

[23] Scolt A. Guelcher, Yuri E. Solomentsev, Paul J. Sides, Anderson J.L., J. Electrochem. Soc., 145 (1998), 1848.
Received 2016-06-22 Accepted 2016-12-09 